Marktqualität und Insiderhandel auf Kapitalmärkten 

Gernot Hinterleitner • Philipp Hornung

\section{Marktqualität und \\ Insiderhandel auf Kapitalmärkten}

Eine experimentelle Untersuchung unterschiedlicher Handelsformen

说 Springer Gabler 
Gernot Hinterleitner

Graz, Österreich
Philipp Hornung

Steyr, Österreich

Die Arbeit wurde im Juli 2011 von der Fakultät für Sozial- und Wirtschaftswissenschaften der Karl-Franzens-Universität Graz als Dissertation angenommen.

Die Autoren danken der Karl-Franzens-Universität Graz für die Unterstützung durch die Gewährung des Förderungsstipendiums und des Forschungsstipendiums sowie für die Verleihung des Maximilian-Jung-Preises für besonders innovative Forschungsarbeiten.

ISBN 978-3-658-12849-4

ISBN 978-3-658-12850-0 (eBook)

DOI 10.1007/978-3-658-12850-0

Die Deutsche Nationalbibliothek verzeichnet diese Publikation in der Deutschen Nationalbibliografie; detaillierte bibliografische Daten sind im Internet über http://dnb.d-nb.de abrufbar.

Springer Gabler

(C) Springer Fachmedien Wiesbaden 2016

Das Werk einschließlich aller seiner Teile ist urheberrechtlich geschützt. Jede Verwertung, die nicht ausdrücklich vom Urheberrechtsgesetz zugelassen ist, bedarf der vorherigen Zustimmung des Verlags. Das gilt insbesondere für Vervielfältigungen, Bearbeitungen, Übersetzungen, Mikroverfilmungen und die Einspeicherung und Verarbeitung in elektronischen Systemen.

Die Wiedergabe von Gebrauchsnamen, Handelsnamen, Warenbezeichnungen usw. in diesem Werk berechtigt auch ohne besondere Kennzeichnung nicht zu der Annahme, dass solche Namen im Sinne der Warenzeichen- und Markenschutz-Gesetzgebung als frei zu betrachten wären und daher von jedermann benutzt werden dürften.

Der Verlag, die Autoren und die Herausgeber gehen davon aus, dass die Angaben und Informationen in diesem Werk zum Zeitpunkt der Veröffentlichung vollständig und korrekt sind. Weder der Verlag noch die Autoren oder die Herausgeber übernehmen, ausdrücklich oder implizit, Gewähr für den Inhalt des Werkes, etwaige Fehler oder Äußerungen.

Gedruckt auf säurefreiem und chlorfrei gebleichtem Papier

Springer Gabler ist Teil von Springer Nature

Die eingetragene Gesellschaft ist Springer Fachmedien Wiesbaden GmbH 
Unseren Eltern 



\section{Inhalt}

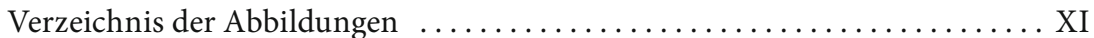

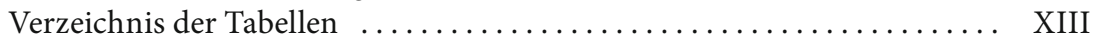

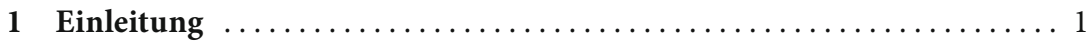

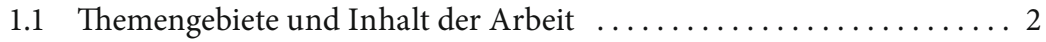

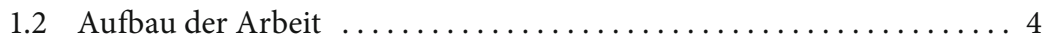

2 Handelsformen an realen Wertpapierbörsen $\ldots \ldots \ldots \ldots \ldots \ldots \ldots 7$

2.1 Eigenschaften und Gestaltungsmöglichkeiten der Call Auction . . . 8 8

2.1.1 Markttransparenz in der Pre-Trading-Phase ........... 10

2.1.2 Regeln und Methoden der Preisbestimmung und der Auftragsrationierung $\ldots \ldots \ldots \ldots \ldots \ldots \ldots \ldots \ldots \ldots$

2.1.3 Weitere Gestaltungsmerkmale von Call Auctions ........ 16

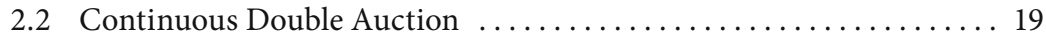

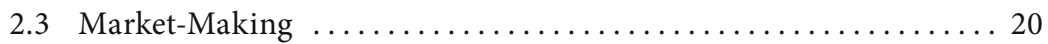

2.4 Mischformen und Ergänzungsmärkte $\ldots \ldots \ldots \ldots \ldots \ldots \ldots \ldots \ldots \ldots \ldots \ldots$

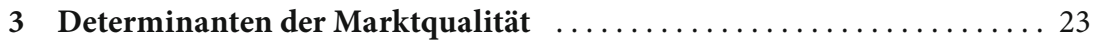

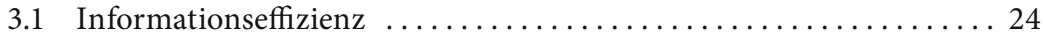

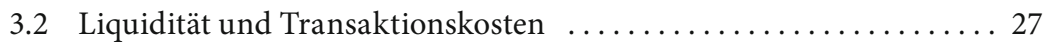

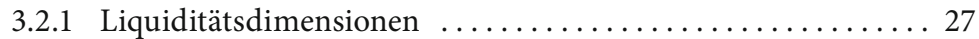

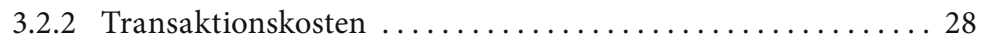

3.2.2.1 Ausführungskosten . .................... 29

3.2.2.2 Explizite Transaktionskosten $\ldots \ldots \ldots \ldots \ldots \ldots \ldots$

3.2 .3 Bid-Ask-Spread und Liquidität $\ldots \ldots \ldots \ldots \ldots \ldots \ldots \ldots \ldots \ldots$

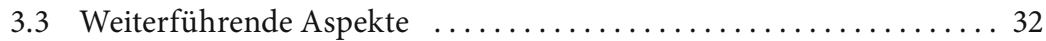


3.3.1 Permanente und transitorische Kursschwankungen ...... 34

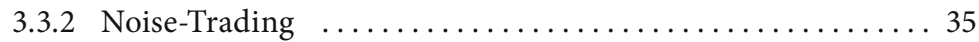

4 Handelsformen und Marktqualität - Literaturüberblick ... . . . . . 39

4.1 Analysen der Einzelhandelsformen $\ldots \ldots \ldots \ldots \ldots \ldots \ldots \ldots \ldots$

4.1.1 Theoretische Analysen ................... 40

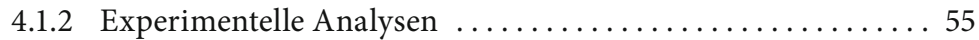

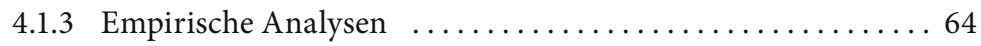

4.2 Market-Opening und Handelsformen $\ldots \ldots \ldots \ldots \ldots \ldots \ldots$

4.2.1 Marktqualität zur Handelseröffnung .............. 70

4.2.2 Opening und Pre-Opening: Marktformenvergleich . . . . . . 76

4.2.3 Eröffnende Call Auction und kontinuierlicher

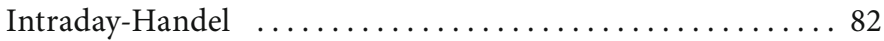

4.2.3.1 NASDAQ und NYSE $\ldots \ldots \ldots \ldots \ldots \ldots \ldots \ldots \ldots$

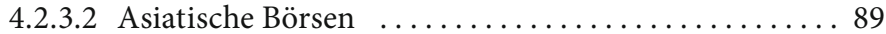

4.2.3.3 Euronext Paris und Deutsche Börse ........... 94

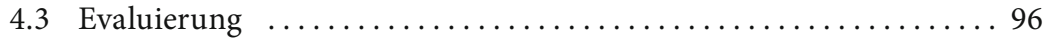

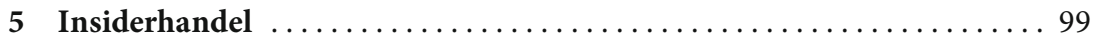

5.1 Definition der Begriffe Insider und Insiderhandel $\ldots \ldots \ldots \ldots \ldots 100$

5.2 Allgemeine Auswirkungen von Insiderhandel . . . . . . . . . . . 104

5.3 Auswirkungen von Insiderhandel auf die Informationseffizienz $\ldots 107$

6 Insiderhandel - Literaturüberblick $\ldots \ldots \ldots \ldots \ldots \ldots \ldots \ldots \ldots \ldots$

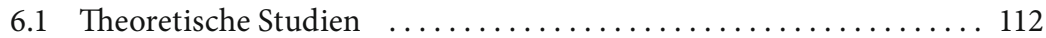

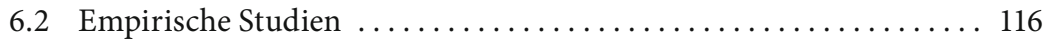

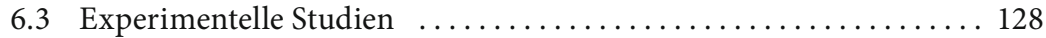

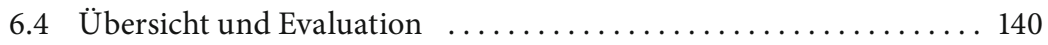

7 Die Fragestellungen der beiden Untersuchungsgebiete $\ldots \ldots \ldots \ldots \ldots 147$

8 Experimente in der Kapitalmarktforschung und Wahl einer

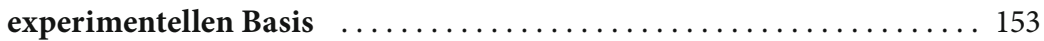

8.1 Bedeutung der experimentellen Wirtschaftsforschung . . . . . . 153

8.2 Vorteilhaftigkeit experimenteller Kapitalmarktanalysen . . . . . . 156

8.3 Anforderungen an das Experimentdesign $\ldots \ldots \ldots \ldots \ldots \ldots \ldots 158$

8.4 Wahl einer experimentellen Basis $\ldots \ldots \ldots \ldots \ldots \ldots \ldots \ldots \ldots$ 


\section{Aufbau, Gestaltung und Durchführung des}

Kapitalmarktexperiments ........................... 167

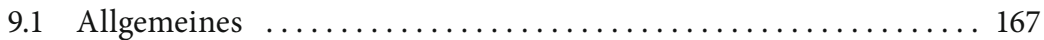

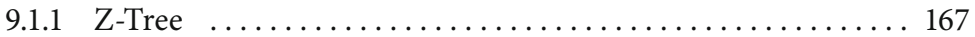

9.1.2 Teilnehmerpool $\ldots \ldots \ldots \ldots \ldots \ldots \ldots \ldots \ldots \ldots \ldots \ldots . \ldots \ldots$

9.1.3 Zeit und Ort der Experimentdurchführung ........... 169

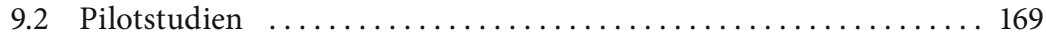

9.2.1 Marktgröße und erste Pilotstudie $\ldots \ldots \ldots \ldots \ldots \ldots \ldots$

9.2.2 Preissignalspannweite und zweite Pilotstudie $\ldots \ldots \ldots \ldots . . \ldots 170$

9.2.3 Marktstruktur und dritte Pilotstudie $\ldots \ldots \ldots \ldots \ldots \ldots . \ldots 171$

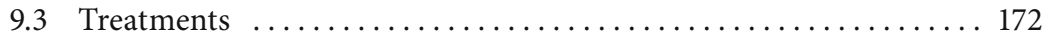

9.3.1 Treatment 1 - Continuous Double Auction (CDA) ...... 172

9.3.2 Subtreatment - Insiderhandel .................. 177

9.3.3 Treatment 2 - Ergänzungsmarkt mit intransparenter Call Auction (EM) ......................... 181

9.3.4 Treatment 3 - Ergänzungsmarkt mit transparenter

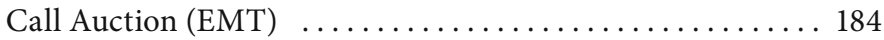

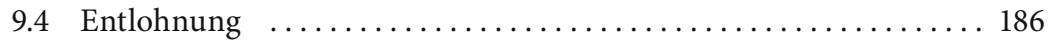

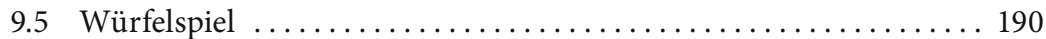

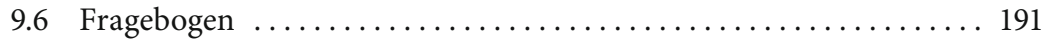

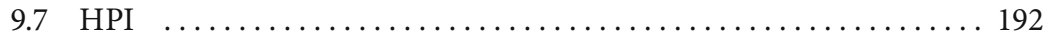

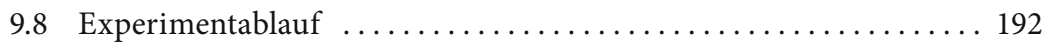

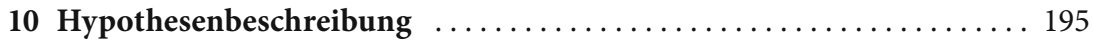

10.1 Marktqualität und Eröffnungsauktion $\ldots \ldots \ldots \ldots \ldots \ldots \ldots \ldots$

10.1.1 Pre-Opening-Phase und Markteröffnung $\ldots \ldots \ldots \ldots \ldots 195$

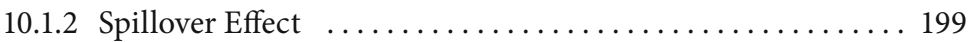

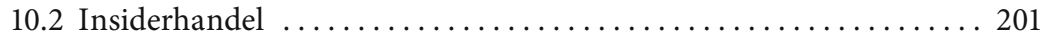

10.2.1 Verhalten, Strategien und Ordererteilung $\ldots \ldots \ldots \ldots 202$

10.2.2 Gewinnerzielung und -verteilung ................ 204

10.2.3 Einfluss auf die Marktqualität ................. 206

10.2.4 Bestrafung, Ehrlichkeit und geschlechtsspezifische Unterschiede .......................... 207

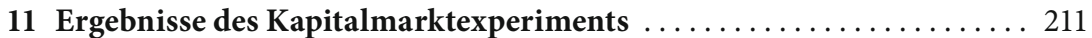

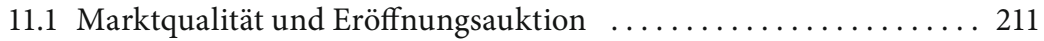

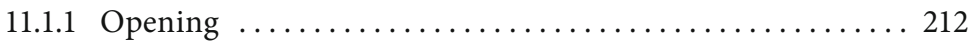

11.1.2 Pre-Opening: Preisentwicklung und Orderflow ........ 220

11.1.3 Spillover Effect $\ldots \ldots \ldots \ldots \ldots \ldots \ldots \ldots \ldots \ldots \ldots \ldots \ldots \ldots$ 


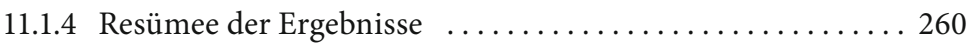

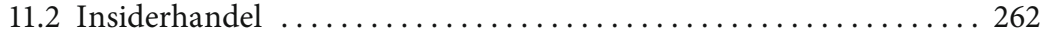

11.2.1 Verhalten, Strategien und Ordererteilung . . . . . . . 262

11.2.2 Gewinnerzielung und -verteilung ............ 278

11.2.3 Einfluss auf die Marktqualität $\ldots \ldots \ldots \ldots \ldots \ldots \ldots \ldots \ldots$

11.2.4 Bestrafung, Ehrlichkeit und geschlechtsspezifische

Unterschiede ............................. 290

\section{Zusammenfassung der Ergebnisse und Ansätze für weitere}

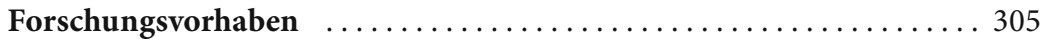

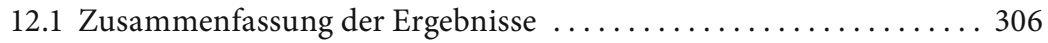

12.2 Ansätze für weitere Forschungsvorhaben . . . . . . . . . . . . . 309

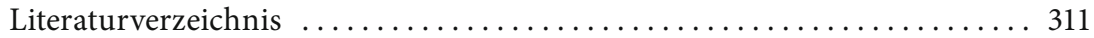

Anhang - Instruktion, Selbsttest und Fragebogen $\ldots \ldots \ldots \ldots \ldots \ldots$

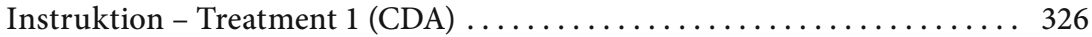

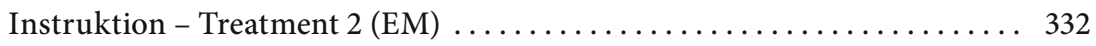

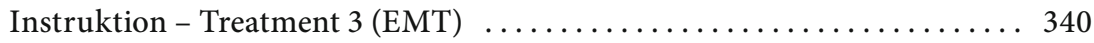

Instruktion - Insiderhandel $(\mathrm{CDA}, \mathrm{EM}, \mathrm{EMT}) \ldots \ldots \ldots \ldots \ldots \ldots \ldots$

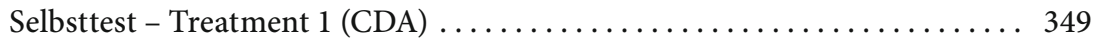

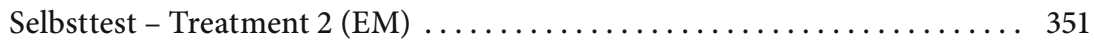

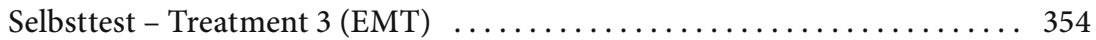

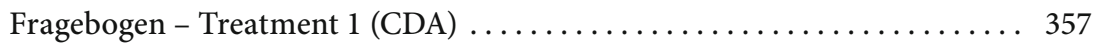

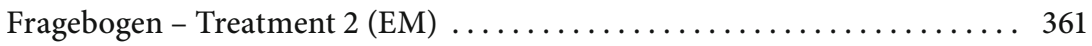

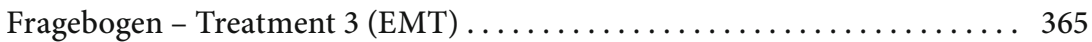




\section{Verzeichnis der Abbildungen}

Abb. 1 Grundstruktur des täglichen Börsenhandels. ............. 3

Abb. 2 Spreads zu Beginn des kont. Handels (Euronext Paris und Xetra) . 95

Abb. 3 Handelsbildschirm der kontinuierlichen Auktion ........... 175

Abb. 4 Die erste Entscheidung im Subtreatment ................ 178

Abb. 5 Die zweite Entscheidung im Subtreatment $\ldots \ldots \ldots \ldots \ldots \ldots . \ldots 179$

Abb. 6 Handelsbildschirm der intransparenten Call Auction ........ 182

Abb. 7 Ergebnisbildschirm der Call Auction $\ldots \ldots \ldots \ldots \ldots \ldots \ldots \ldots . \ldots \ldots$

Abb. 8 Einordnung der Transparenzniveaus $\ldots \ldots \ldots \ldots \ldots \ldots \ldots \ldots$

Abb. 9 Handelsbildschirm der transparenten Call Auction .......... 185

Abb. 10 Die Entlohnungsschemata im Vergleich ................. 189

Abb. 11 Experimentablauf .............................. 194

Abb. 12 Boxplots des MRE der unterschiedlichen Opening-

Mechanismen ................................ 213

Abb. 13 Stück-Handelsvolumen in den beiden Call Auctions . ......... 218

Abb. 14 MRE-Werte über Pre-Opening-Phase in der intransparenten

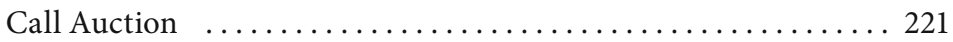

Abb. 15 MRE-Werte über Pre-Opening-Phase in der transparenten

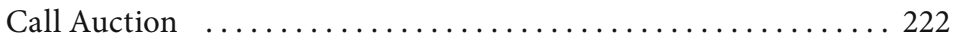

Abb. 16 Volatilität der indikativen Preise in den beiden Call Auctions . . 225

Abb. 17 Durchschnittl. kumuliertes Brutto-Auftragsvolumen über

Pre-Opening-Phase .......................... 226

Abb. 18 Durchschnittl. kumuliertes Netto-Auftragsvolumen über Pre-

Opening-Phase ............................. 227

Abb. 19 Durchschnittliche Stornierungsquoten in der Pre-OpeningPhase .................................... 230

Abb. 20 Bruttoauftragsvolumen und MRE-Werte - Transparente

Auktion 
Abb. 21 Indikative Preise und Fundamentalwert - Durchlauf 1,

Periode 2 ..................................... 237

Abb. 22 Preissignale der Handelsteilnehmer - Durchlauf 1, Periode 2 . . . 238

Abb. 23 Preisverlauf in der CDA des EM - Durchlauf vom 26.11.2009,

Periode 4 ................................... 244

Abb. 24 Preisverlauf in der CDA des EMT - Durchlauf vom 27.4.2010,

Periode 4 .................................... 244

Abb. 25 Preisverlauf in der reinen CDA - Durchlauf vom 22.10.2009,

Periode 4 .................................... 245

Abb. 26 Stück-Handelsvolumen in den kontinuierlichen Auktionen … 252

Abb. 27 Zusammenhang MRE Opening und MRE in der CDA des EM

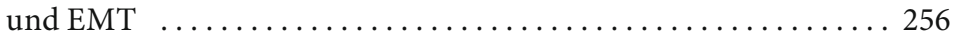

Abb. 28 Manipulationsversuch in der transp. Call Auction,

Durchlauf 17, Periode 10 ....................... 268

Abb. 29 Manipulationsversuch in der transp. Call Auction,

Durchlauf 19, Periode 8 .............................. 269

Abb. 30 Häufigkeitsverteilung der Ordergrößen der Insider . . . . . . . . . 276

Abb. 31 Häufigkeitsverteilung der Ordergrößen der Nicht-Insider . . . . . 277

Abb. 32 Gewinnverteilung - Insider ...................... 278

Abb. 33 Gewinnverteilung - Nicht Insider .................... 279

Abb. 34 Gewinne in Abhängigkeit der Anzahl der Insider .......... 281

Abb. 35 MRE in Abhängigkeit der Anzahl der Insider ............. 287

Abb. 36 Spreads in Abhängigkeit der Anzahl der Insider ............. 289

Abb. 37 Anteil der Entscheidungen in Abhängigkeit der Aufdeckungsw. . 291

Abb. 38 Gewinnverteilung der weiblichen Insider ............... 295

Abb. 39 Gewinnverteilung der männlichen Insider ............... 295

Abb. 40 Häufigkeiten - Augenzahlen beim Würfelspiel - alle

Teilnehmer ..................................... 298

Abb. 41 Häufigkeiten - Augenzahlen beim Würfelspiel - Frauen -

Männer ..................................... 301 


\section{Verzeichnis der Tabellen}

Tab. 1 Preisbildung - Call Auction (vollständige Markträumung) $\ldots \ldots 13$

Tab. 2 Call Auction bei Angebotsüberhang .................. 14

Tab. 3 Mehrere umsatzmaximale Kurse in einer Call Auction ........ 15

Tab. 4 Unterschiedliche Gestaltungsmerkmale von eröffnenden CA

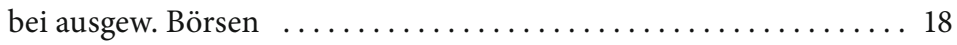

Tab. 5 Kombination CA und CDA am Beispiel der Deutschen Börse .... 22

Tab. 6 Renditen inkl. Overnight-Returns an der NYSE, 1982 . ....... 70

Tab. 7 Kennzahlenvergleich Opening und Closing an der NYSE $\ldots \ldots \ldots 72$

Tab. 8 Eröffnungs- und Schlusskursvolatilitätsrate an der Tel Aviv Stock Exchange. ............................. 77

Tab. 9 Intraday-Renditevolatilitäten vor und nach der Opening-CAIntegration (NASDAQ) $\ldots \ldots \ldots \ldots \ldots \ldots \ldots \ldots \ldots \ldots \ldots \ldots \ldots$

Tab. 10 Veränderung des Pricing-Errors vor und nach Einführung der CA an der SGX ............................ 91

Tab. 11 Übersicht über theoretische Studien bzgl. Insiderhandel $\ldots . \ldots .140$

Tab. 12 Übersicht über empirische Studien bzgl. Insiderhandel . ....... 141

Tab. 13 Übersicht über experimentelle Studien bzgl. Insiderhandel . . . . 142

Tab. 14 Entlohnungsschema des CDA-Treatments ............... 187

Tab. 15 Entlohnungsschema des EM- und des EMT-Treatments $\ldots \ldots \ldots 187$

Tab. 16 Aufschlüsselung der Ausgaben für das Experiment . .......... 190

Tab. 17 Auszahlungsschema des Würfelspiels . ................. 191

Tab. 18 Daten bezüglich der Preissignalstreuungen der 42 Perioden. . . . 211

Tab. 19 Testergebnis - Normalverteilungsannahme der OpeningMRE-Werte ...................................... 214

Tab. 20 Kruskal-Wallis-Test - Unterschied der Eröffnungspreiseffizienz . . 214

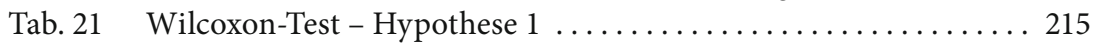

Tab. 22 Wilcoxon-Test - Vergleich der Eröffnungspreiseffizienz der

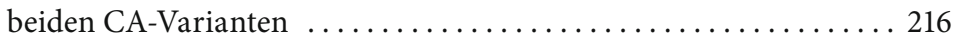


Tab. 23 Spreads in den beiden Call Auctions ..................... 219

Tab. 24 Wilcoxon-Test - Liquidität in den beiden Call Auctions . ....... 219

Tab. 25 Friedman-Test - MRE-Unterschiede während des

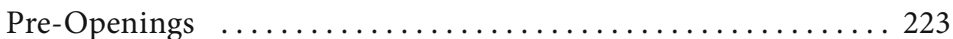

Tab. 26 Wilcoxon-Test - Unterschiede zwischen den MRE-Werten

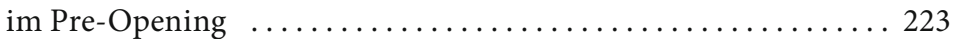

Tab. 27 Wilcoxon-Test - Effizienz-Unterschiede im Pre-Opening zwischen den beiden CA ............................ 224

Tab. 28 Wilcoxon-Test - Unterschiede der Pre-Opening-Volatilität . . . . 225

Tab. 29 Wilcoxon-Test - Unterschiede in den Stornierungsquoten ..... 231

Tab. 30 Regression - Einfluss Stornierungsquoten ............... 233

Tab. 31 Korrelation - MRE nach 60 Sek. und BAV in der 2. Hälfte

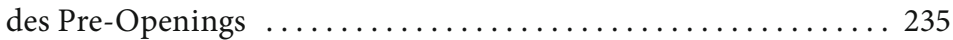

Tab. 32 Korrelation - Opening-MRE und BAV in der 2. Hälfte des

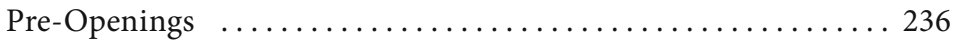

Tab. 33 MRE-Werte in der kontinuierlichen Auktion ............. 246

Tab. 34 Kruskal-Wallis ANOVA - Unterschiede der MRE-Werte . ..... 247

Tab. 35 Wilcoxon-Test - Unterschiede der durchschnittlichen Effizienz .................................... 247

Tab. 36 Wilcoxon-Test - Unterschiede der Informationseffizienz . . . . . . 248

Tab. 37 Durchschnittliche Bid-Ask-Spreads in der kontinuierlichen Auktion ..................................... 249

Tab. 38 Kruskal-Wallis-Test - Spread-Unterschiede ................ 249

Tab. 39 Wilcoxon-Test - Spread-Unterschiede CDA vs. EM und EMT . . 250

Tab. 40 Wilcoxon-Test - Spread-Unterschiede zwischen kont. Auktionen ................................... 250

Tab. 41 Kruskal-Wallis-Test - Unterschiede des StückHandelsvolumens ............................... 252

Tab. 42 Wilcoxon-Test - Unterschiede des Stückhandelsvolumens $\ldots . .252$

Tab. 43 Intraday-Renditevolatilität der Treatmens .............. 253

Tab. 44 Einfaktorielle ANOVA auf Unterschiede der IntradayVolatilität ................................. 254

Tab. 45 Wilcoxon-Test - Paarweiser Vergleich der Intraday-Volatilität . . 254

Tab. 46 Regression - Einfluss des Opening-Preises ............... 256

Tab. 47 Regression - MRE Opening und Volatilität in der kontinuierlichen Auktion ....................... 257

Tab. 48 Regression - MRE Opening und Spreads in der kontinuierlichen Auktion ......................... 257

Tab. 49 Anzahl der Insider in den Treatments .................. 262 
Tab. 50 Kruskal-Wallis-Test - Insider je Periode $\ldots \ldots \ldots \ldots \ldots \ldots \ldots 262$

Tab. 51 Mann-Whitney-Test - Insider je Periode ................ 263

Tab. 52 Aufdeckungswahrscheinlichkeiten bei Annahme des Insiderstatus ..................................... 264

Tab. 53 Chi-Quadrat-Test - Unterschiede Annahme des Insiderstatus . . . 264

Tab. 54 Kruskal-Wallis-Test - Aufdeckungswahrsch. bei Annahme

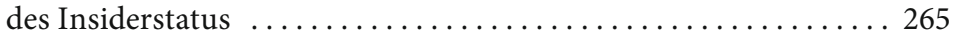

Tab. 55 Angezeigte Aufdeckungswahrsch. vor Entscheidung bzgl. des Insiderstatus . ............................... 265

Tab. 56 Chi-Quadrat-Test - Aufdeckungswahrsch. vor Entsch. bzgl. des Insiderstatus ............................... 265

Tab. 57 T-Test für unabhängige Stichproben - HPI-R des EM- und des EMT-Marktes ............................... 266

Tab. 58 Anzahl der von den beiden Händlertypen erstellten ersten

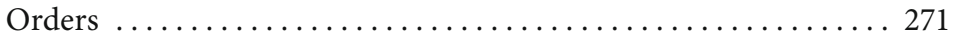

Tab. 59 Chi-Quadrat-Test - Ordererstellung in der reinen CDA $\ldots \ldots \ldots 271$

Tab. 60 Chi-Quadrat-Test - Ordererstellung im EM-Treatment . ...... 272

Tab. 61 Chi-Quadrat-Test - Ordererstellung im EMT-Treatment ...... 272

Tab. 62 Anzahl der erstellten Orders je Quartil der CDA . . . . ....... 273

Tab. 63 Friedman-Test - Insideraktivität in der reinen CDA . . . . . . . 273

Tab. 64 Friedman-Test - Insideraktivität in der CDA des EM . . ....... 274

Tab. 65 Friedman-Test nsideraktivität in der CDA des EMT . . . . . . . 274

Tab. 66 Anzahl der durchgeführten Orders je Quartil der CDA ....... 275

Tab. 67 Wilcoxon-Test auf Unterschiede der Orderdurchführbarkeit . . . 275

Tab. 68 Mann-Whitney-Test - Unterschiede der Ordergrößen Insider - Nicht Insider . ........................ 277

Tab. 69 Mann-Whitney-Test - Unterschiede der Gewinne Insider - Nicht Insider . . . . . . . . . . . . . . . . . . . . . . . 279

Tab. 70 Regression - Insidergewinne - Anzahl Insider ............. 282

Tab. 71 Regression - Gewinne der Nicht-Insider - Anzahl Insider … 282

Tab. 72 Wilcoxon-Test - Kauf- und Verkauforders zur

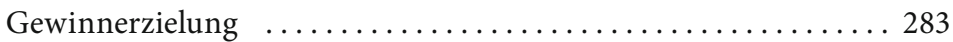

Tab. 73 Wilcoxon-Test - Gewinne in CA/CDA des EM ............ 284

Tab. 74 Wilcoxon-Test - Gewinne in CA/CDA des EMT ........... 284

Tab. 75 Korrelationsergebnis - HPI-C - Ergebnis ............... 285

Tab. 76 Regression - MRE der CDA des EM - Anzahl Insider . ........ 287

Tab. 77 Regression - Spreads der CDA des EMT - Anzahl Insider . . . . . 289

Tab. 78 Wilcoxon-Test - Unterschiede der Aufdeckungswahrsch. Annahme - Ablehnung .......................... 291 
Tab. 79 Wilcoxon-Test - max. Aufdeckungsw. bei Ann. - min. Aufdeckungsw. bei Abl. . . . . . . . . . .................. 293

Tab. 80 Mittelwerte der Aufdeckungsw. - Frauen - Männer . . . . . . . . 293

Tab. 81 T-Test - max. Aufdeckungsw. bei Ann. - min. Aufdeckungsw. bei Abl. ........................................... 294

Tab. 82 Mann-Whitney-Test - Unterschiede Gewinne weibliche und männliche Insider .............................. 296

Tab. 83 Ergebnisse des Würfelspiels und Interesse an Insiderhandel alle Teilnehmer .................................. 297

Tab. 84 Chi-Quadrat-Test - Gleichverteilung der Augenzahlen $\ldots . \ldots . .298$

Tab. 85 Geschlechtsspezifisches Interesse an Insiderstatus . . . . . . . . 299

Tab. 86 Ergebnisse des Würfelspiels und Interesse an Insiderhandel Frauen ...................................... 300

Tab. 87 Ergebnisse des Würfelspiels und Interesse an Insiderhandel -

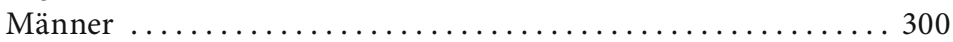

Tab. 88 Mann-Whitney-Test - Insiderinteresse $\ldots \ldots \ldots \ldots \ldots \ldots \ldots \ldots 2$

Tab. 89 Mann-Whitney-Test - Augenzahl ..................... 302

Tab. 90 Kreuztabelle - Geschlecht - Augenzahl ................ 302

Tab. 91 Chi-Quadrat-Test - Geschlecht - Augenzahl ............. 302

Tab. 92 Korrelationsergebnis - HPI-R - minimale Aufdeckungswahrscheinlichkeit .................... 304 